\title{
Influência da Educação Musical Avançada na Discriminação Auditiva com Ruído Competitivo
}

\section{Influence of Advanced Music Education on Speech Discrimination with Competitive}

\section{Noise}

\author{
David Tomé ${ }^{1,2^{*}}$, Ana Elisa Dias ${ }^{1}$, Ilídio Pereira ${ }^{3}$ \\ 'Laboratório de Audiologia, Escola Superior de Saúde do Politécnico do Porto (ESS-P.Porto), Porto, Portugal \\ ${ }^{2}$ Neurocognition Group, Laboratório de Reabilitação Psicossocial (LabRP), CiR, Porto, Portugal \\ ${ }^{3}$ Área Técnico-Científica de Biomatemática, Bioestatística e Bioinformática, Escola Superior de Saúde do Politécnico do Porto (ESS-P.Porto), Porto, Portugal \\ *凶dts@eu.ipp.pt
}

\section{Resumo}

Introdução: A organização neuronal para a música e linguagem tem sido alvo de inúmeras discussões nas áreas de audiologia, neurociência e arte musical. Vários estudos têm procurado investigar a influência da prática musical na discriminação da fala com ruído competitivo. Objetivos: Averiguar se a prática musical frequente promove uma melhoria significativa na capacidade de discriminação tonal e vocal em ambientes ruidosos. Material e métodos: 15 estudantes de música com prática instrumental (GE) e 15 não-estudantes de música (GC) realizaram medições audiológicas de discriminação de tons puros e de fala com ruído competitivo. Todos os participantes foram submetidos a um teste de discriminação de tons puros, o teste de mudanças de limiar determinado modificado (TMLDm), e outro de discriminação de fala (TMLDm vocal), ambos na presença de ruído competitivo. Resultados: Todos os participantes eram normouvintes e tinham idades entre os 16 e os 33 anos ( $G E, M=19.1$ anos, $D P=4.2 ; G C, M=20.1, D P=5.4)$. $O G E$ superou significativamente o GC em ambos os testes (valor $p<0.05$ ). Tanto no ouvido direito como no esquerdo no TMLDm tonal o GE obteve uma média da relação sinal-ruído de $-5 \mathrm{~dB}$. No TMLDm vocal, os participantes do GC erraram em média a repetição de 8 palavras perante a média de 1 falha do GE. Conclusões: Os resultados sugerem que a prática musical pode melhorar o desempenho na discriminação da palavra em ruído competitivo, o que permite igualmente antever um melhor processamento auditivo central.

Palavras-chave: Prática musical, pseudo palavras, ruído, discriminação, processamento auditivo.

\begin{abstract}
Summary
Introduction: The neuronal organization for music and language has been the subject of numerous discussions in the areas of audiology, neuroscience and musical art. Several studies have sought to investigate the influence of musical practice on speech discrimination with competitive noise. Objectives: To ascertain whether the frequent musical practice promotes a significant improvement in the capacity of tone and speech discrimination in noisy environments. Material and methods: 15 music students with instrumental practice (GE) and 15 non-music students (GC) performed hearing discrimination testing with pure tones and speech with competitive noise. All participants underwent a pure tone discrimination test, the modified changing threshold test (TMLDm), and speech discrimination test (speech TMLDm), both with competitive noise. Results: All participants were normal-hearing and aged between 16 and 33 years old ( $G E, M=19.1$ years, $S D=4.2 ; G C, M=20.1, S D=5.4$ ). The $G E$ significantly exceeded the $C G$ in both tests ( $p$ value $<0.05$ ). In both the right and left ears in tone TMLDm, the GE obtained a mean signal-to-noise ratio of $-5 \mathrm{~dB}$. In the speech TMLDm, the participants of the CG erred, on average, the repetition of 8 words compared to the average of 1 failure of the GE. Conclusions: The results suggest that musical practice can improve performance in word discrimination with competitive noise, which also allows foreseeing better central auditory processing.
\end{abstract}

Keywords: Musical practice, pseudowords, noise, discrimination, auditory processing.

\section{INTRODUÇÃO}

Ouvir depende da capacidade biológica inata e da experiência do indivíduo no meio ambiente (Campiotto et al., 1997). Porém, raramente somos confrontados com uma única mensagem auditiva, mas antes com a ocorrência simultânea de sinais acústicos complexos que o sistema auditivo deve processar para descodificar a informação contida (ParberyClark et al., 2009). Um dos melhores exemplos dessa ocorrência é ouvir de sinais de fala em contexto de ruído competitivo. A compreensão da fala é um dos requisitos fundamentais para o desenvolvimento da comunicação humana, a sua importância e relação com a capacidade auditiva foi reconhecida há mais de 
um século por Oscar Wolf, que considerou a fala como "a forma mais perfeita para testar o poder da audição, já que a mesma incorpora as nuances mais delicadas de frequência, intensidade $e$ característica do som" (Musiek \& Rintelmann, 2001).

Em 1996 a American Speech-Language Hearing Association (ASHA), definiu processamento auditivo central como sendo os mecanismos e processos do sistema auditivo responsáveis pelos seguintes fenómenos comportamentais: localização e lateralização sonora; discriminação auditiva; reconhecimento de padrões auditivos; aspectos temporais da audição, incluindo resolução temporal, mascaramento temporal, integração temporal e ordenação temporal; desempenho auditivo na presença de sinais competitivos e desempenho auditivo com sinais acústicos degradados. Estes mecanismos estão previstos tanto para sons verbais como não-verbais, estando envolvidos diversos sistemas e processos neurocognitivos (ASHA, 1996; Phillips, 1995; Musiek \& Rintelmann, 2001).

Parece existir uma ténue barreira entre o processamento auditivo e a discriminação da fala, se assim for, pessoas com treino auditivo poderão discriminar melhor as mensagens verbais, como por exemplo músicos. Segundo Reid \& Holland (2008), os músicos estando habituados a usar e explorar a sua audição, têm mais facilidade em identificar as características específicas de um determinado som. Alguns estudos já demonstraram a influência da prática musical na melhoria de algumas faculdades individuais, como a atenção visual (Rodrigues et al., 2006) e a cognição (Galvão, 2006). Estas descobertas poderão ir ao encontro de estudos neuroanatómicos e imagiológicos que compararam cérebros de músicos e de não músicos, apresentando os do primeiro grupo uma maior quantidade de massa cinzenta e maior atividade funcional via ressonância magnética funcional (RMNf) (Platel et al., 1997; Schneider et al., 2002) em estruturas como o corpo caloso (Schlaug et al., 2005; Schmithorst \& Wilke, 2002), o volume da matéria cinzenta do giro de Heschl (Gaser \& Schlaug, 2003; Schneider et al., 2002), o lobo temporal (Keenan et al., 2001; Luders et al., 2004; Schlaug et al., 2005), o giro frontal inferior (Gaser \& Schlaug, 2003; Luders et al., 2004), o córtice motor primário (Amunts et al., 1997) e o cerebelo (Hutchinsons et al., 2003). Verificou-se também uma similaridade nas estruturas que são activadas pelo processamento da música e pelo processamento da fala (Brown et al., 2004; Levitin \& Menon, 2003). Com base nestes dados, deduz-se que, se a prática musical influencia a organização anátomo-funcional do cérebro, e se existem similaridades neurofuncionais nalguns aspectos de neuroprocessamento de música e de linguagem, a prática musical poderá influenciar positivamente o neurodesenvolviemnto da linguagem (Besson et al., 2007).

Na música e na fala existe um processamento para a frequência e ritmo associados a processos neurocognitivos de alta especialização cortical onde estão associados processos de atenção e memória (Muszkat et al., 2000).

As pistas perceptuais são importantes para 0 processamento auditivo. Deste modo, ouvintes com melhores capacidades perceptuais auditivas podem detectar pistas acústicas mais subtis, estando mais aptos a dissociar o sinal de referência do ruído de fundo. É o que ocorre, por exemplo, nas situações de conversação em ambientes ruidosos (Caporali et al., 2004). Esta ideia é reforçada por pesquisas que apontam para o facto de a atenção e a memória modularem a percepção da fala em ruído. Henrich et al. (2008) concluiu que a extracção do sinal de fala de ruído de fundo baseia-se em recursos atencionais. A necessidade dessa mesma extracção resultará numa menor disponibilidade de memória auditiva a curto prazo para retenção, repetição e lembrança do sinal vocal. Essas descobertas apontam para o facto de os indivíduos com memória mais desenvolvida poderem sentir-se menos afectados por ruído de fundo e apresentarem melhores recursos atencionais, que se reflectem num melhor desempenho em tarefas de discriminação de fala com ruído competitivo. É o que aponta o estudo desenvolvido por Parbery-Clark et al. (2009), conclui que as capacidades desenvolvidas pela prática musical se transferem-se para a fala. Os resultados sugerem que se deve a um maior desenvolvimento da memória e discriminação frequencial por parte dos músicos. Essa diferença (em relação ao grupo de controlo) era ainda mais evidente quando as pistas de localização espacial se encontravam neutralizadas (a fala e o ruído provinham da mesma fonte sonora).

O treino auditivo proporcionado pela prática musical apresenta-se como um fator que exerce influência na capacidade de reconhecer a fala no silêncio e no ruído. No silêncio, músicos e não músicos apresentam desempenhos semelhantes. Porém, em tarefas de reconhecimento de frases apresentadas com ruído competitivo, os músicos apresentam melhores desempenhos, indicando que a prática musical é uma atividade que melhora a capacidade de reconhecimento da fala, quando esta ocorre em ambientes ruidosos (Soncini \& Costa, 2006).

Música e fala, embora sejam sinais complexos, correspondem a diferentes tipos de estimulação auditiva. Tanto o código musical como o código da fala possuem diferentes elementos e vias. Isto porque a fala possui frequentemente um significado denotativo, enquanto o código utilizado na música não separa significante e significado, uma vez que a mensagem da música não está condicionada a convenções semântico-linguísticas. A mensagem da música consiste antes numa organização sonora que traduz ideias por uma estrutura significativa que é a própria mensagem: a música (Muszkat et al., 2000; Pederiva \& Tristão, 2006). Assim, surge a questão se a prática musical proporciona uma melhor discriminação de tons puros mas também de pseudo-palavras, com ruído competitivo.

Este estudo tem como objetivo verificar se a prática musical promove uma melhoria significativa na capacidade de discriminação tonal e vocal em ambientes ruidosos, visto que a comunicação oral, em situação real, não se encontra desprovida de contexto.

Neste aspeto, o presente artigo tem carácter inovador, dado a amostra ser constituída por jovens-adultos, ainda estudantes, e também ao facto de se tratar da população portuguesa.

\section{MATERIAL E MÉTODOS}

\subsection{AMOSTRA}

Este é um estudo observacional analítico e descritivo, tem como objetivo verificar se a prática musical permite desenvolver capacidades de processamento auditivo no sentido de otimizar a competência de discriminação de tons puros e pseudo- 
palavras em ambientes ruidosos.

O grupo de controlo foi constituído por 15 participantes ( 5 género masculino, idade compreendida [17-33] anos; $M=20.1$ anos; $D P=5.4$ ), todos com nacionalidade portuguesa e residentes na área metropolitana do Porto. Os quinze elementos não tinham qualquer formação na área da Música (excepto a disciplina de Educação Musical de $5^{\circ}$ e $6^{\circ}$ anos, integrada no plano nacional de escolaridade obrigatória), nem qualquer prática musical.

Do grupo experimental, fizeram parte igualmente 15 participantes (5 género masculino, idade [17-32]; $M=19.1$ anos; $\mathrm{DP}=4.2$ ), de nacionalidade portuguesa, e residentes na área metropolitana do Porto. Frequentam o Conservatório de Música do Porto, e foram seleccionados de entre os que tinham prática de estudo instrumental regular há pelo menos 8 anos de estudo. Todos os participantes praticam 1 a 2 instrumentos, sendo o tempo médio de estudo diário de 2 horas e 20 minutos.

Em todos os participantes verificaram-se os seguintes critérios de inclusão: ausência de sintomas ou queixas de vertigens, tonturas, enxaquecas, perda de equilíbrio e instabilidade postural; ausência de sinais clínicos sugestivos de patologia vestibular ou neurológica; sem história clínica de hipertrofia das adenóides e disfunção da trompa de Eustáquio; sem história clínica ou sinais de otites recorrentes, pelo menos nos últimos 6 meses. E como critério de exclusão, a existência de perda auditiva (de acordo com as normas do B.I.A.P., 2004) aquando da recolha dos dados.

Este estudo foi autorizado pelas instituições intervenientes, tendo todos os participantes assinado o consentimento informado.

\subsection{PROCEDIMENTOS}

O tom puro é um parâmetro musical importante, uma vez que permite definir aspectos melódicos de uma sequência musical. Como parâmetro básico dos sons, o tom torna-se tanto musical como linguisticamente relevante (Vance, 1976). Num estudo desenvolvido por Besson et al. (2007), concluiu-se que a prática musical melhora quer o processamento da fala, quer o processamento dos tons. Para verificar se o processamento de tons puros melhora a par do processamento de estímulos vocais (pseudo-palavras), decidiu-se adaptar o teste de Mudanças de Limiar Determinado (TMLD) por meio de mascaramento. O TMLD envolve a apresentação binaural de um tom pulsátil, a uma frequência específica, ou de palavras, enquanto o ruído mascarador é apresentado bilateralmente (Musiek \& Rintelmann, 2001). A modificação do teste MLD, doravante TMLDm, consistiu na estimulação monoaural e no varrimento frequencial utilizado na audiometria tonal convencional, com apresentação de ruído de banda estreita ipsilateralmente, $50 \mathrm{~dB}$ acima do limiar tonal (frequência a frequência), registando-se as mudanças de limiar auditivo na presença de ruído competitivo. O mesmo procedimento foi realizado com estímulos vocais (pseudo-palavras). Uma melhor relação sinal-ruído (S-R) significa que o participante processa o sinal em condições acústicas menos favoráveis e, por conseguinte, tem um melhor processamento auditivo central de tons puros.

Em todos os participantes o protocolo de exames iniciou-se com a obtenção do consentimento informado, seguido de anamnese, otoscopia, timpanometria a $226 \mathrm{~Hz}$, audiometria tonal (obtenção do limiar auditivo) e audiometria vocal (SRT e percentagem de discriminação). Seguidamente, aplicou-se o TMLDm tonal, que consistiu na apresentação monoaural de ruído de banda estreita, $50 \mathrm{~dB}$ acima do limiar tonal (frequência a frequência) determinado anteriormente. A partir daqui, procedeu-se como numa audiometria tonal convencional, método ascendente, apresentando estímulos de tom puro pulsáteis ipsilateralmente com o ruído, de modo a determinar novo limiar auditivo e assim obter a relação sinalruído (S-R). Por último, realizou-se a prova vocal, em que foram apresentadas monoauralmente 20 pseudo-palavras, escolhidas aleatoriamente (das listas de Lafon), $40 \mathrm{~dB}$ acima do SRT, com speech noise ipsilateral, numa relação sinal-ruído de $0 \mathrm{~dB}$.

\subsection{RESULTADOS}

Pela aplicação do teste à normalidade, assumiu-se que os dados obtidos em ambos os ouvidos no TMLDm vocal e tonal nas frequências 250,500, 1000, 2000, 4000 e $8000 \mathrm{~Hz}$ seguem a distribuição binomial. A frequência de $125 \mathrm{~Hz}$ revelou-se de difícil avaliação e obtenção por parte dos participantes, sendo por isso excluída da análise estatística. Aplicou-se o teste não paramétrico correspondente para amostras independentes (Mann-Whitney). Foram obtidos resultados significativos (valor $p<0.05$ ) no TMLDm tonal em ambas as estimulações monoaurais (ouvido direito e esquerdo) para todas as frequências testadas, excepto a $8000 \mathrm{~Hz}$ no ouvido direito (Tabela 1). Também no TMLDm vocal com pseudo-palavras, em ambas as estimulações monoaurais (ouvido direito e esquerdo) foram obtidos resultados estatisticamente significativos, com o grupo de controlo a ter cerca de 4 repetições erradas para cada ouvido e o grupo de músicos com apenas 1 erro para o ouvido esquerdo e 0 erros para o ouvido direito (Tabela 2).

\section{DISCUSSÃO}

Os resultados obtidos apontam para o facto de os indivíduos com prática musical regular apresentarem um melhor desempenho na avaliação da discriminação de tons puros e de fala na presença de ruído competitivo.

NoTMLDm tonal, apenas o resultado da frequência de 8000 $\mathrm{Hz}$ no ouvido direito não revelou uma diferença significativa na relação S-R entre os dois grupos. No entanto, devido ao tamanho reduzido da amostra, não é possível ser concludente quanto a esta constatação. Em todas as frequências do ouvido esquerdo, e também nas restantes frequências do ouvido direito, se evidenciou uma melhor performance do grupo de músicos (experimental), com resultados uniformes entre si (medianas entre -10 e $-5 \mathrm{~dB}$ ).

No TMLDm vocal, as diferenças entre os dois grupos também foram evidentes. Os indivíduos do grupo de controlo falharam, em média, um total de 8 palavras perante a média de 1 falha do grupo experimental. Por ser uma amostra demasiadamente pequena, não foi possível estabelecer relações entre o número de instrumentos, o número de anos de estudo e o tempo dispendido diariamente a tocar com o desempenho na avaliação efectuada. Os resultados deste estudo são consistentes com a suposição de Besson et al. (2007), que refere que a prática musical regular poderá influenciar de forma 
positiva alguns aspectos do processamento da linguagem.

Estão igualmente em concordância com a literatura que suporta a existência de mecanismos comuns no processamento da linguagem e da música (Steinbeis \& Koelsch, 2008; Brown et al., 2004; Levitin \& Menon, 2003), e que constata que o treino auditivo realizado para um tipo de estímulo sonoro pode ser generalizado para outros estímulos ou situações de escuta não utilizados nas situações de treino (Oxenham et al., 2003).

De um modo geral, as funções musicais são complexas, múltiplas e de localizações assimétricas, envolvendo o hemisfério direito para altura, percepção espacial, timbre e discriminação melódica, e o hemisfério esquerdo para ritmos, identificação da semântica musical, senso de familiaridade, processamento temporal e sequencial dos sons. As alterações fisiológicas induzidas pela estimulação sonora podem reflectirse nas mudanças de padrões, no reflexo de orientação, na variabilidade das respostas fisiológicas envolvidas em processos de atenção e expectativas musicais ou na mudança de frequência, topografia e amplitude dos ritmos eléctricos cerebrais (Muszkat \& Correia, 2000; Tervaniemi et al. 2005).

Os músicos treinam horas seguidas, estando concentrados na manipulação de complexos sinais auditivos, que incluem múltiplos processamentos das três características do sinal acústico (intensidade, frequência e tempo). Além de processar unidades auditivas concomitantes entre si (isto é, a ocorrência simultânea de diferentes melodias), devem analisar a relação vertical entre os diferentes processos (harmonia). Mais ainda, os músicos apuram as suas capacidades de modo a compreender, planear e tocar em tempo real. A prática musical não só desenvolve aspectos específicos da percepção musical, mas também outros domínios, particularmente a linguagem, o que sugere mecanismos comuns no processamento de linguagem e de música (Steinbeis \& Koelsch, 2008).

A atenção e a memória modelam a percepção da fala em ruído, isto é, indivíduos com memória auditiva a curto prazo mais desenvolvida podem sentir-se menos afectados por ruído de fundo e ter melhores recursos atencionais, que se irão reflectir num melhor desempenho em tarefas de discriminação de fala com ruído competitivo (Henrich et al., 2008; Zatorre et al., 1994). Com base nessa informação e nos resultados deste trabalho, pode supor-se que a prática musical desenvolve a memória auditiva a curto prazo bem como os recursos atencionais. Esta dedução vai ao encontro da conclusão de Parbery-Clark et al. (2009) no estudo que desenvolveram, referindo maior desenvolvimento da memória verificado no grupo de músicos e o facto de as capacidades desenvolvidas pela prática musical se transferirem para a fala.

Existe também compatibilidade com os resultados obtidos no estudo desenvolvido por Soncini \& Costa (2006), em que os músicos apresentaram melhores desempenhos no reconhecimento da fala em ambientes ruidosos.

Considerando todos os aspectos abordados e analisados, foi possível observar que a prática musical regular favorece a discriminação tonal e vocal, em situações em que a condição de escuta é desfavorável. Logo, a prática musical tem subentendido o desenvolvimento de capacidades superiores no processamento auditivo central, de entre as quais poderão estar a memória auditiva a curto prazo e os recursos atencionais, nomeadamente a capacidade de concentração, importante para a aquisição de novas aprendizagens. Tornou-se assim evidente que o treino auditivo realizado para um determinado tipo de estimulação (a música) pode promover melhorias nas várias vertentes do processamento auditivo.

Para estudos futuros, seria interessante avaliar uma amostra de maiores dimensões, de modo a poder estabelecer relações entre os resultados e o número de instrumentos praticados, o número de anos de estudo, tempo de prática diária e até mesmo a idade de início de estudo musical. Seria igualmente construtivo perceber a diferença existente no desempenho de testes com ruído competitivo utilizando listas de palavras e listas de frases.

Tabela 1: Resultados obtidos no Teste de Mudanças de Limiar Determinado modificado, com estimulação tonal nas frequências $250,500,1000,2000$, 4000 e $8000 \mathrm{~Hz}$

\begin{tabular}{|c|c|c|c|c|c|c|c|}
\hline \multirow[t]{2}{*}{ Frequência } & \multirow[t]{2}{*}{ Grupo } & \multicolumn{2}{|c|}{ Mediana (S-R) } & \multicolumn{2}{|c|}{$\begin{array}{c}\text { Desvio } \\
\text { Interquartis }\end{array}$} & \multicolumn{2}{|c|}{ Valor Prova } \\
\hline & & OD & OE & OD & $\mathrm{OE}$ & OD & $\mathrm{OE}$ \\
\hline \multirow{2}{*}{$250 \mathrm{~Hz}$} & Experimental & -10 & -10 & 2.5 & 5 & \multirow{2}{*}{0.004} & \multirow{2}{*}{0.016} \\
\hline & Controlo & 0 & 0 & 5 & 5 & & \\
\hline \multirow{2}{*}{$500 \mathrm{~Hz}$} & Experimental & -5 & -10 & 5 & 5 & \multirow{2}{*}{0.041} & \multirow{2}{*}{0.015} \\
\hline & Controlo & 0 & 0 & 5 & 7.5 & & \\
\hline \multirow{2}{*}{$1000 \mathrm{~Hz}$} & Experimental & -10 & -10 & 5 & 7.5 & \multirow{2}{*}{0.002} & \multirow{2}{*}{0.006} \\
\hline & Controlo & 0 & 0 & 2.5 & 5 & & \\
\hline \multirow{2}{*}{$2000 \mathrm{~Hz}$} & Experimental & -5 & -5 & 5 & 5 & \multirow{2}{*}{0.018} & \multirow{2}{*}{0.007} \\
\hline & Controlo & -5 & 0 & 5 & 2.5 & & \\
\hline \multirow{2}{*}{$4000 \mathrm{~Hz}$} & Experimental & -10 & -10 & 2.5 & 2.5 & \multirow{2}{*}{0.004} & \multirow{2}{*}{0.003} \\
\hline & Controlo & 0 & 0 & 5 & 5 & & \\
\hline \multirow{2}{*}{$8000 \mathrm{~Hz}$} & Experimental & 0 & -10 & 5 & 7.5 & \multirow{2}{*}{0.164} & \multirow{2}{*}{0.003} \\
\hline & Controlo & 0 & 0 & 5 & 2.5 & & \\
\hline
\end{tabular}

OD: ouvido direito; OE: ouvido esquerdo; S-R: diferença sinal-ruído; Valor prova a negrito $<0.05$ (a). 
Artigos Científicos

Tabela 2: Resultados obtidos no TMLDm vocal (pseudo-palavras)

\begin{tabular}{|c|c|c|c|c|}
\hline Ouvido & Grupo & Mediana & $\begin{array}{c}\text { Desvio } \\
\text { Interquartis }\end{array}$ & Valor Prova \\
\hline \multirow{2}{*}{ OD } & Experimental & 0 & 1.0 & \multirow{2}{*}{0.000} \\
\hline & Controlo & 4 & 0.5 & \\
\hline \multirow{2}{*}{$\mathrm{OE}$} & Experimental & 1 & 0.5 & \multirow{2}{*}{0.000} \\
\hline & Controlo & 4 & 0.5 & \\
\hline \multirow{2}{*}{$\begin{array}{c}\text { Total } \\
(\mathrm{OD}+\mathrm{OE})\end{array}$} & Experimental & 1 & 1.5 & \multirow{2}{*}{0.000} \\
\hline & Controlo & 8 & 1.0 & \\
\hline
\end{tabular}

OD: ouvido direito; OE: ouvido esquerdo; Valor prova a negrito $<0.05$ (a).

\section{CONCLUSÕES}

Aprender e praticar música foi sempre considerado importante e valorizado por todas as áreas que estudam as capacidades cognitivas do Ser Humano. No entanto, só nas últimas décadas se tem objetivado e mensurado com diferentes metodologias os diversos benefícios da prática de uma arte tão ancestral como é a música.

Os resultados obtidos neste estudo vão de encontro à literatura existente, uma vez que evidenciam a melhor performance dos estudantes de música (em relação a nãoestudantes de música) em provas standard de discriminação de tons puros e de pseudo-palavras com ruído competitivo. Foram encontradas diferenças estatisticamente significativas tanto no número de falhas nas pseudo-palavras, como nas relações sinalruído, entre os dois grupos amostrais. Sugerindo que a prática musical regular permite melhorar a discriminação auditiva em ambientes ruidosos.

Visando uma hipotética aplicabilidade, estas conclusões podem incentivar a utilização da música como recurso terapêutico para situações de alteração do processamento central da mensagem auditiva ou como forma de prevenção.

Dado o interesse da associação entre a aprendizagem musical e o desenvolvimento de estruturas cerebrais que se traduzem numa maior eficácia na discriminação auditiva, demonstrado nesta pesquisa, espera-se que o presente trabalho seja, futuramente, impulsionador de outros estudos complementares, uma vez que em Portugal a investigação neste âmbito é ainda muito escassa.

\section{REFERÊNCIAS BIBLIOGRÁFICAS}

American Speech-Language-Hearing Association. Central auditory processing current status of research and implications for clinical practice. Am J of Audiology 5(2):41-54, 1996.

Amunts K. Motor cortex and hand motor skills: Structural compliance in the human brain. Human Brain Mapp 5:206-215, 1997.

Besson M, Schön D, Moreno S, Santos A, Magne C. Influence of musical expertise and musical training on pitch processing in music and language. Restor Neurol Neurosci. 25:399-410, 2007.

Bureau International D'Audiophonologie (BIAP, 2004). Audiometric classification of hearing impairment - recommendation 02/1.

Brown S, Parsons LM, Martinez MJ, Hodges DA, Fox PT. The song system of the human brain. Cogn Brain Res 20:363-375, 2004.

Campiotto AR, Levy C, Holzheim D, Rabinovich K, Vicente LCC, Cartiglioni
M, Redondo MC, Anelli W. Tratado de fonoaudiologia. São Paulo: Editora Roca Ltda. 1997.

Caporali SA, Silva JA. Reconhecimento de fala no ruído em jovens e idosos com perda auditiva. BJORL 70(4):525-32, 2004.

Galvão A. Cognição, emoção e expertise musical. Psicologia: Teoria e Pesquisa 22(2):169-174, 2006.

Gaser C, Schlaug G. Gray matter differences between musicians and nonmusicians. Ann N Y Acad Sci 999:514-517, 2003.

Henrich J. (2008) A cultural species. In: Explaining culture scientifically, ed. Brown, M., pp. 184-210. University of Washington Press.

Hutchinson S, Lee LHL, Gaab N, Schlaug G. Cerebellar volume of musicians. Cereb Cortex 13:943-949, 2003.

Keenan JP, Thangaraj V, Halpern AR, Schlaug G. Absolute pitch and planum temporale. Neuroimage 14:1402-1408, 2001.

Levitin DJ, Menon V. Musical structure is processed in 'language' areas of the brain: a possible role for Brodmann Area 47 in temporal coherence. Neuroimage 20:2142-2152, 2003.

Luders E, Gaser C, Jancke L, Schlaug G. A voxel-based approach to gray matter asymmetries. Neuroimage 22:656-664, 2004.

Oxenham AJ, Fligor BJ, Mason CR, Kidd G Jr. Informational masking and musical training. J Acoust Soc Am 114(3):1543-1549, 2003.

Parbery-Clark A, Skoe E, Lam C, Kraus N. Musician enhancement for speech-in-noise. Ear \& Hearing 30(6):653-661, 2009.

Pederiva PLM, Tristão RM. Música e cognição. Ciências \& Cognição 9:8390, 2006.

Phillips DP. Central Auditory Processing: a view from auditory neuroscience. Am J Otology 16:338-352, 1995.

Platel H, Price C, Baron JC, Wise R, Lambert J, Frackowiak RSJ, Lechevalier $B$, Eustache J. The structural components of music perception. A functional anatomical study. Brain 120:229-43, 1997.

Musiek FE, Rintelmann WF. Perspectivas actuais em avaliação auditiva. 1a Edição. Brasil: Editora Manole Ltda.; 2001.

Muszkat M, Correia CMF, Campos SM. Música e neurociências. Revista Neurociências 8(2):70-75, 2000.

Reid AW, Holland MW. Ouvir o som. Reino Unido: Associação de Orquestras Britânicas, 2008.

Rodrigues ACO, Guerra LB, Loureiro MA. Música e cognição: a atenção visual é diferente em músicos e não-músicos? Congresso da Associação Nacional de Pesquisa e Pós-graduação em Música; Brasília, 2006.

Schlaug G, Norton A, Overy K, Winner E. Effects of music training on the child's brain and cognitive development. Ann NY Acad Sci 1060:219-230, 2005.

Schmithorst VJ, Wilke M. Differences in white matter architecture between musicians and non-musicians: a diffusion tensor imaging study. Neurosci Lett 321:57-60, 2002. 


\section{Artigos Científicos}

Schneider P, Scherg M, Dosch HG, Specht HJ, Gutschalk A, Rupp A. Morphology of Heschl's gyrus reflects enhanced activation in the auditory cortex of musicians. Nat Neurosci 5:688-694, 2002.

Soncini F, Costa MJ. Efeito da prática musical no reconhecimento da $\mathrm{f}$ ala no silêncio e no ruído. Pró-Fono Revista de Actualização Científica 18(2):161-170, 2006.

Steinbeis N, Koelsch S. Shared neural resources between music and language indicate semantic processing of musical tensionresolution patterns. Cereb Cortex 18(5):1169-1178, 2008.

Tervaniemi M, Just V, Koelsch S, Widmann A, Schröger E. Pitch discrimination accuracy in musicians vs nonmusicians: an eventrelated potential and behavioral. Exp Brain Res 161:1-10, 2005.
Vance TJ. An experimental investigation of tone and intonation in Cantonese. Phonetica 33:368-392, 1976.

Zatorre RJ, Evans AC, Meyer E. Neural mechanisms underlying melodic perception and memory for pitch. J Neurosci 14:1908-19, 1994.

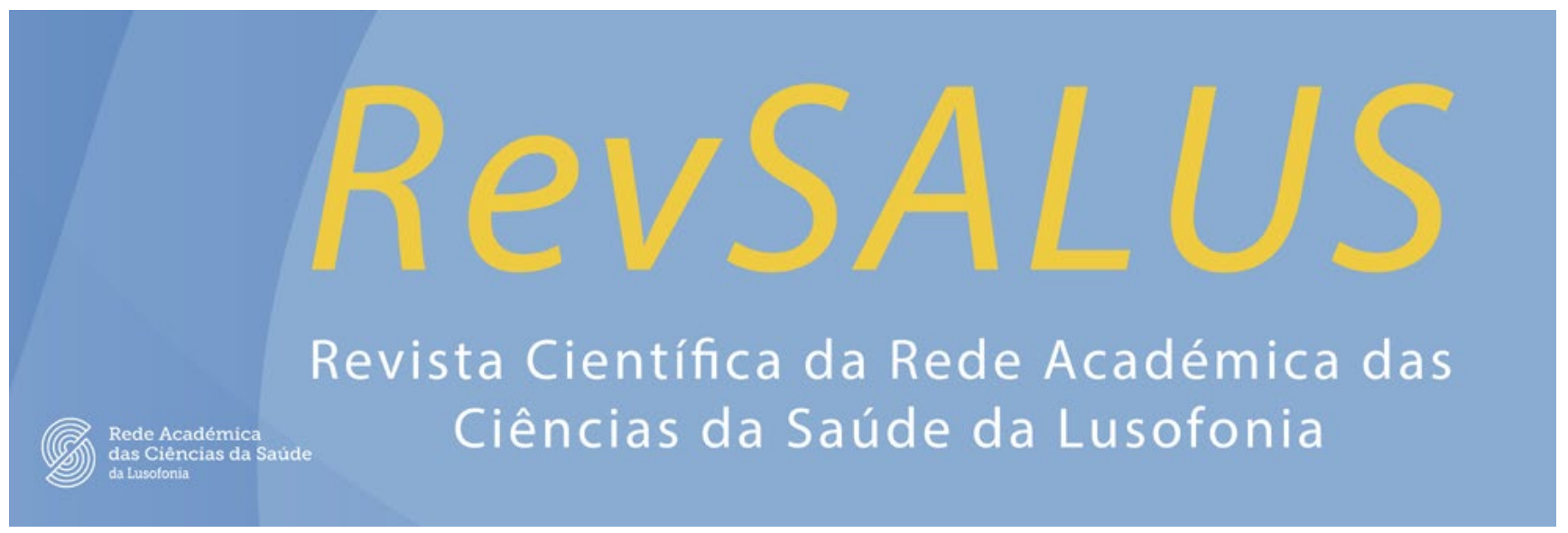

